seen how broadly the approach to retinal progenitor cell differentiation by the WNT pathway modulation can be applied to differentiation of other tissue or cell types. Nevertheless, predifferentiation of ESCs in vitro to a desired cell population before transplantation both to ensure efficiency of transplantation as well as to minimize the risk of tumor formation may be a good general strategy in stem cell therapy.

\section{Acknowledgments}

This work was supported by 973 Program grants (2013CB967504); NSFC grants (no. 81270992 and no. 81130017); NEI/NIH grants EY014428, EY018660, EY019270, EY021374; VA Merit Award; and the Burroughs Wellcome Fund Clinical Scientist Award in Translational Research.

Address correspondence to: Yehong Zhuo, State Key Laboratory of Ophthalmology, Zhongshan Ophthalmic Center,
Sun Yat-sen University, 54 South Xianlie Road, Guangzhou, 510060, China. Phone: 86.20.87330371; Fax: 86.20.87333271; E-mail: zhuoyh@mail.sysu.edu.cn. Or to: Kang Zhang, Institute for Genomic Medicine, University of California, San Diego, Department of Ophthalmology and Shiley Eye Center, 9415 Campus Point Drive, La Jolla, California 92093-0946, USA. Phone: 858.246.0814; Fax: 858.246.0873; E-mail: kang.zhang@gmail.com.

1. Evans MJ, Kaufman MH. Establishment in culture of pluripotential cells from mouse embryos. Nature. 1981;292(5819):154-156.

2. Doi $\mathrm{D}$, et al. Prolonged maturation culture favors a reduction in the tumorigenicity and the dopaminergic function of human ESC-derived neural cells in a primate model of Parkinson's disease. Stem Cells. 2012;30(5):935-945.

3. Lamba DA, Gust J, Reh TA. Transplantation of human embryonic stem cell-derived photoreceptors restores some visual function in Crx-deficient mice. Cell Stem Cell. 2009;4(1):73-79.

4. Schwartz SD, et al. Embryonic stem cell trials for macular degeneration: a preliminary report. Lancet. 2012;379(9817):713-720.
5. Cui L, et al. WNT signaling determines tumorigenicity and function of ESC-derived retinal progenitors. J Clin Invest. 2013;123(4):1647-1661.

6. Przyborski SA. Differentiation of human embryonic stem cells after transplantation in immune-deficient mice. Stem Cells. 2005;23(9):1242-1250.

7. Kielman MF, et al. Apc modulates embryonic stem-cell differentiation by controlling the dosage of beta-catenin signaling. Nat Genet. 2002; 32(4):594-605

8. Meyer JS, et al. Modeling early retinal development with human embryonic and induced pluripotent stem cells. Proc Natl Acad Sci U S A. 2009; 106(39):16698-16703

9. Osakada F, Ooto S, Akagi T, Mandai M, Akaike A, Takahashi $\mathrm{M}$. Wnt signaling promotes regeneration in the retina of adult mammals. J Neurosci. 2007; 27(15):4210-4219.

10. Zhang K, Ding S. Stem cells and eye development. NEng J Med. 2011;365(4):370-372.

11. Hannum G, et al. Genome-wide methylation profiles reveal quantitative views of human aging rates. Mol Cell. 2013;49(2):359-367.

12. Li W, et al. Rapid induction and long-term self-renewal of primitive neural precursors from human embryonic stem cells by small molecule inhibitors. Proc Natl Acad Sci U S A. 2011;108(20):8299-8304.

13. West EL, et al. Defining the integration capacity of embryonic stem cell-derived photoreceptor precursors. Stem Cells. 2012;30(7):1424-1435.

\title{
Striking the target in iron overload disorders
}

\author{
Karin E. Finberg
}

Department of Pathology, Duke University Medical Center, Durham, North Carolina, USA.

\begin{abstract}
The liver, a major site of body iron stores, mediates key responses that preserve systemic iron homeostasis. In this issue of the JCI, Guo et al. demonstrate that administration of antisense oligonucleotides that reduce expression of Tmprss6, a hepatic protein that plays an essential role in maintaining iron balance, can attenuate disease severity in mouse models of human iron overload disorders. These data reveal the potential of novel TMPRSS6-targeted therapies for the treatment of clinical conditions such as hereditary hemochromatosis and $\beta$-thalassemia.
\end{abstract}

\section{Hepcidin and the regulation of systemic iron balance}

The majority of iron required daily by the adult human body is used to meet the demands of hemoglobin synthesis. Most of this iron is obtained through the recycling of senescent erythrocytes by macrophages in the spleen, liver, and bone marrow, while a small amount is absorbed from the diet in the duodenum. Hepcidin, a small circulating peptide released by the liver, regulates iron balance by limiting both the absorption of iron from the diet and the release of iron from macrophage stores (1).

Conflict of interest: The author has declared that no conflict of interest exists.

Citation for this article: J Clin Invest. 2013; 123(4):1424-1427. doi:10.1172/JCI68889.
Hepcidin mediates these effects by triggerferroportin, a cellular iron exporter that is highly expressed at the basolateral membrane of enterocytes and the cell membrane of macrophages (Figure 1). In hepatocytes, hepcidin transcription is modulated by an intracellular signaling cascade that is activated by binding of BMP ligands to a cellsurface receptor complex (Figure 2). The liver, a major site of iron storage, increases production of the BMP family member BMP6 in response to rising local iron stores; this leads to increased signaling for hepcidin production, which in turn limits further dietary iron absorption. Appropriate regulation of intestinal iron absorption is critical, as there is no regulated mechanism for eliminating surplus iron from the body. ing the internalization and degradation of

\section{Hepcidin insufficiency in iron overload disorders}

Inherited forms of iron overload (hemochromatosis) result from mutations in gene products that are required locally in the liver for hepcidin production. In these disorders, the resulting hepcidin insufficiency leads to gastrointestinal iron absorption that exceeds the body's needs. The accumulation of excess iron promotes oxidative damage to tissues, which can ultimately lead to failure of organs such as the heart, liver, and endocrine glands. Hepcidin levels are inappropriately low relative to body iron stores in another class of clinical disorders associated with systemic iron loading: congenital anemias that are characterized by ineffective erythropoiesis (IE) (2). IE describes a defective form of erythroid maturation characterized by an increased proportion of erythroid precursors, which, due to excessive apoptosis, fail to produce the normal complement of mature erythrocytes. In $\beta$-thalassemia, the most common inherited form of IE, the primary genetic defect leads to reduced synthesis of the $\beta$-globin component of adult hemoglobin. The result is an excess 


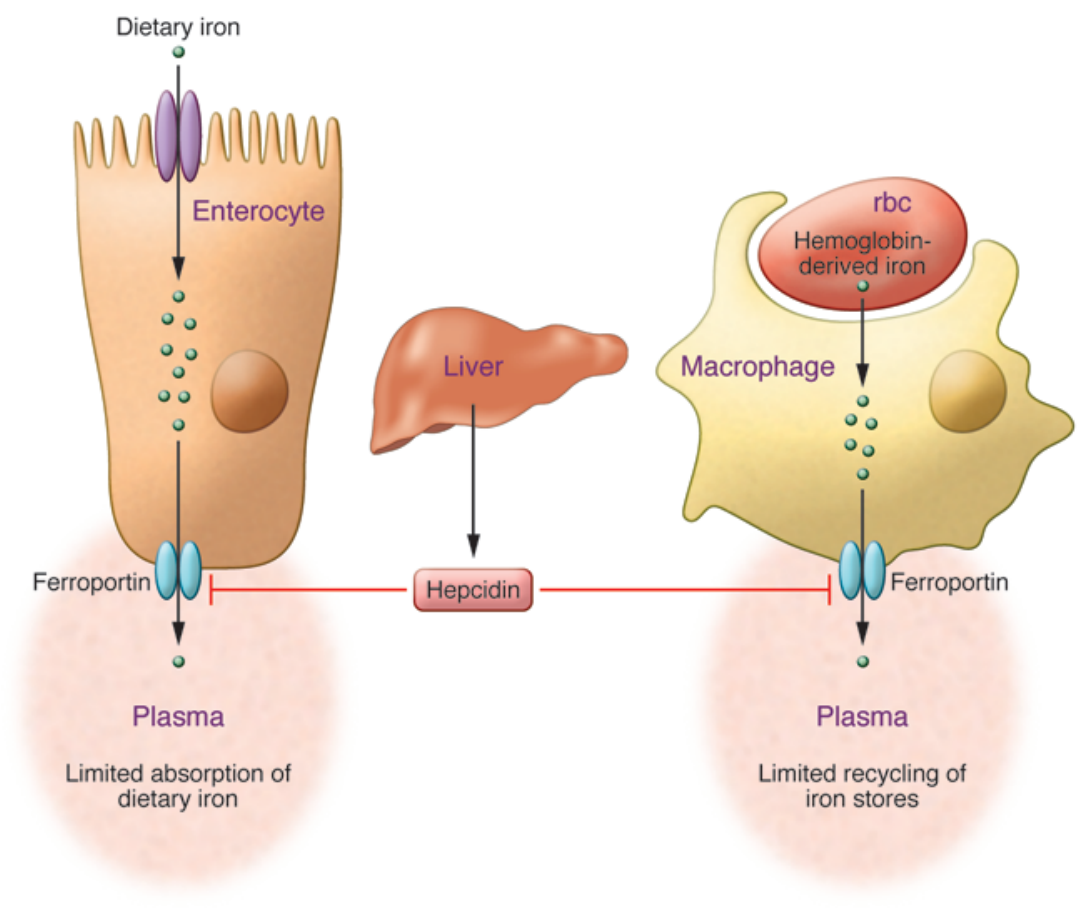

of unpaired $\alpha$-globin chains; these form toxic aggregates that promote apoptosis of erythroid precursors. Patients with $\beta$-thalassemia major, the most severe form of the disease, develop iron overload from blood transfusions that are required to sustain life. Iron overload also develops in patients with $\beta$-thalassemia intermedia (TI), who by definition are not transfusion dependent, due to the hepcidin suppression associated with IE. In these patients, the hepcidin response increases iron availability for erythropoiesis but is also maladaptive, as it promotes the development of systemic iron overload. The molecular basis by which IE leads to hepcidin suppression is not yet understood.

Transgenic overexpression of hepcidin has been shown to limit iron loading in a mouse model of TI (3) and to prevent hepatic iron overload in a mouse model of $H F E$-associated hemochromatosis (HFE$\mathrm{HH}$ ), the most common inherited form of hemochromatosis resulting from mutation in the HFE gene (4). Additionally, chronic injection of synthetic hepcidin lowered plasma iron levels in the HFE$\mathrm{HH}$ mouse model (5). However, the bioactive form of hepcidin shows rapid renal excretion, and disulfide bridging within the molecule makes chemical synthesis expensive. These limitations have spurred the development of small-peptide hepcidin mimetics (termed "minihepcidins"), which have shown early therapeutic promise in mouse models (6). In this issue of the JCI, Guo et al. demonstrate an alternative pharmacological strategy that addresses the hepcidin insufficiency in iron overload disorders by increasing endogenous hepcidin production (7).

\section{Tmprss 6 as a therapeutic target}

In the liver, hepcidin synthesis through BMP signaling involves a number of extracellular, membrane-bound, and intracellular proteins (Figure 2). A key negative regulator of hepcidin synthesis by this pathway is TMPRSS6 (also known as matriptase-2), a transmembrane protein primarily expressed in the liver (8). TMPRSS6, which contains a serine protease domain, is thought to downregulate BMP signaling by cleaving a membrane-associated protein termed hemojuvelin (HJV), which functions as a BMP coreceptor, from the cell surface (9). Accordingly, both humans and mice with TMPRSS6 mutations exhibit inappropriately elevated levels of hepcidin, leading to impaired dietary iron absorption, systemic iron deficiency, and iron deficiency anemia. The effects of Tmprss 6 disruption are thus similar to those obtained by transgenic overexpression of hepcidin, and targeted genetic disruption of Tmprss 6 can reduce iron loading in mouse models of HFE-HH (10) and TI (11).

\section{Figure 1}

Effect of hepcidin on iron transport in the duodenum and in macrophages. (Left) After dietary iron is taken up by duodenal enterocytes, ferroportin mediates iron export across the basolateral membrane into the plasma. (Right) After red blood cells are phagocytosed by macrophages, hemoglobin-derived iron is exported by ferroportin into the plasma. In both of these cell types, hepcidin limits iron export by triggering the internalization and degradation of ferroportin in lysosomes.
To induce endogenous hepcidin expression, Guo et al. developed antisense oligonucleotides (ASOs) that target Tmprss6 mRNA (7). ASOs are single-stranded, chemically modified nucleic acid analogs, which following Watson-Crick base pairing, induce selective degradation of their target mRNA by the natural enzyme RNase H (12). Of over 150 ASOs designed to target murine Tmprss6, two were identified that suppressed Tmprss 6 mRNA in a dose-dependent manner when introduced into isolated murine hepatocytes and when injected into healthy mice. Tmprss6ASOs appeared well tolerated, and the treated mice did not show biochemical or histological evidence of hepatic inflammation or injury.

The authors then demonstrated efficacy of Tmprss6-ASOs in mouse models of human iron overload disorders. In a model of HFE-HH, biweekly Tmprss6-ASO injections for six weeks raised hepcidin expression and reduced iron concentrations in the serum and liver. Tmprss6-ASO treatment also led to sequestration of iron within splenic macrophages, reflecting the ability of hepcidin to limit ferroportinmediated iron export, and to a moderate reduction in blood hemoglobin levels, likely due to the reduced availability of iron for erythropoiesis.

Tmprss-ASOs were also effective in reducing serum and liver iron concentra- 

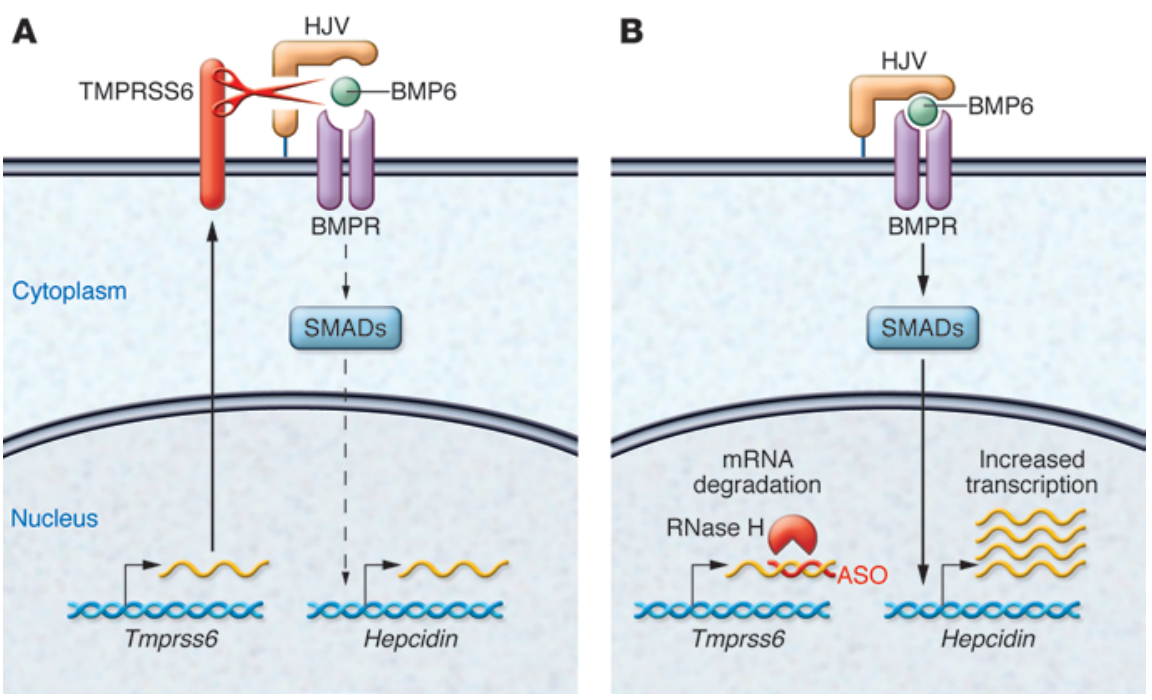

Figure 2

Effect of Tmprss6-ASO on the regulation of hepcidin expression in hepatocytes. (A) Shown in a schematized hepatocyte is the pathway that TMPRSS6 modulates to dampen hepcidin expression. Binding of the BMP6 ligand to BMP receptors (BMPRs) at the plasma membrane activates SMAD proteins, which translocate to the nucleus to activate hepcidin transcription. The membrane-associated protein HJV, which functions as a BMP coreceptor, augments hepcidin signaling through this pathway. TMPRSS6 dampens hepcidin signaling by cleaving HJV from the cell membrane. When liver iron stores increase, BMP6 production by the liver is also increased, thereby inducing hepcidin synthesis. (B) In the presence of Tmprss6-ASOs, Tmprss6 mRNA is degraded by RNase $\mathrm{H}$. The resulting reduction in TMPRSS6 protein leads to enhanced BMP signaling for hepcidin transcription. For simplicity, other hepcidin-modulating pathways, as well as other proteins that interact with the BMP pathway, are not shown.

tions in a mouse model of TI. However, in contrast to findings in the HFE-HH model, Tmprss6-ASO treatment raised hemoglobin levels in the TI mice. This improvement in anemia was accompanied by reductions in erythroid indicators of IE (toxic $\alpha$-globin aggregates, reactive oxygen species, and markers of apoptosis) and a reduction in the splenic enlargement that is characteristic of TI. Notably, similar improvements in erythropoiesis were previously achieved in TI mice through Tmprss6 gene knockout (11), transgenic hepcidin overexpression (3), a low-iron diet (3), and injection of transferrin, the iron-binding protein that delivers iron to erythroid precursors (13). Thus, the mechanism leading to improved erythropoiesis following Tmprss6-ASO treatment, while still uncertain, might relate to the reduced delivery of iron to erythroid precursors.

Recently, Schmidt et al. demonstrated an alternative approach to lower Tmprss6 mRNA in mice based on the principle of RNA interference (14). In that study, a single injection of siRNA formulated in lipid nanoparticles led to hepcidin elevation that persisted for one week. Long-term treatment of mouse models of HFE-HH and TI with Tmprss6-siRNA modified iron homeostasis and erythropoiesis in a manner qualitatively similar to Tmprss6-ASOs.

\section{Looking forward}

In summary, the study of Guo et al. (7), together with that of Schmidt et al. (14), provides exciting proof of principle that TMPRSS6-dependent hepcidin regulation can be exploited as a therapeutic strategy for iron overload disorders characterized by hepcidin insufficiency. Small molecules that selectively inhibit the TMPRSS6 catalytic domain might be predicted to show similar therapeutic effects (15). Pharmacological approaches that effectively target human TMPRSS6 and show acceptable safety profiles would increase management options for iron overload disorders. Currently, patients with HFE-HH with evidence of increased iron stores are treated by long-term phlebotomy (16), a treatment that while safe and effective, is inconvenient and may be limited by poor venous access. Patients with $\beta$-thalassemia, in whom phlebotomy is contraindicated due to preexisting anemia, are treated by pharmacological chelation (17). Treatment with deferoxamine, a subcutaneously administered chelator used extensively in $\beta$-thalassemia, may yield adverse effects such as infusion site reactions and audiologic, ophthalmologic, and bone toxicities. Side effects of deferasirox, a newer oral chelator, include gastrointestinal disturbances, changes in kidney function, and more rarely, hepatic and renal failure.

Whether TMPRSS6-targeted therapies might be applied in isolation or in combination with other therapies remains uncertain. As hepcidin elevation causes a redistribution of iron to macrophages, TMPRSS6-targeted treatments would require careful titration to prevent the development of iron-restricted anemia, particularly if used as an adjunct to phlebotomy in HFE-HH. Given that the availability of oral chelators has improved patient compliance and satisfaction with chelation therapy in $\beta$-thalassemia (17), the acceptance of TMPRSS6-targeted therapies by this patient population might be influenced by the route of administration. As the majority of patients with $\beta$-thalassemia live in low- or middle-income countries, where access to chelation therapy still remains challenging (18), it would be important to consider how these novel therapies could be delivered to the areas of greatest clinical need.

\section{Acknowledgments}

The author thanks Nancy C. Andrews for helpful discussions. K.E. Finberg is supported by NIH K08 DK084204 and by a Burroughs Wellcome Fund Career Award for Medical Scientists.

Address correspondence to: Karin E. Finberg, Duke University Medical Center, Department of Pathology, Box 3712, Durham, North Carolina 27710, USA. Phone: 919.668.0992; Fax 919.681.8461; E-mail: karin.finberg@duke.edu.

1. Ganz T, Nemeth E. Hepcidin and iron homeostasis Biochim Biophys Acta. 2012;1823(9):1434-1443.

2. Ginzburg Y, Rivella S. beta-thalassemia: a model for elucidating the dynamic regulation of ineffective erythropoiesis and iron metabolism. Blood. 2011; 118(16):4321-4330.

3. Gardenghi $S$, et al. Hepcidin as a therapeutic tool to limit iron overload and improve anemia in beta-thalassemic mice. J Clin Invest. 2010; 120(12):4466-4477.

4. Nicolas G, et al. Constitutive hepcidin expression prevents iron overload in a mouse model of hemochromatosis. Nat Genet. 2003;34(1):97-101. 
5. Moran-Jimenez MJ, et al. Hepcidin treatment in $\mathrm{Hfe}^{-/-}$mice diminishes plasma iron without affecting erythropoiesis. Eur J Clin Invest. 2010;40(6):511-517.

6. Preza GC, et al. Minihepcidins are rationally designed small peptides that mimic hepcidin activity in mice and may be useful for the treatment of iron overload. J Clin Invest. 2011;121(12):4880-4888.

7. Guo S, et al. Reducing TMPRSS6 ameliorates hemochromatosis and $\beta$-thalassemia in mice. J Clin Invest. 2013;123(4):1531-1541.

8. Du X, et al. The serine protease TMPRSS6 is required to sense iron deficiency. Science. 2008. 320(5879):1088-1092.

9. Silvestri $\mathrm{L}$, et al. The serine protease matriptase-2 (TMPRSS6) inhibits hepcidin activation by cleaving membrane hemojuvelin. Cell Metab. 2008;
$8(6): 502-511$.

10. Finberg KE, Whittlesey RL, Andrews NC. Tmprss6 is a genetic modifier of the Hfe-hemochromatosis phenotype in mice. Blood. 2011;117(17):4590-4599.

11. Nai A, et al. Deletion of TMPRSS6 attenuates the phenotype in a mouse model of beta-thalassemia. Blood. 2012;119(21):5021-5029.

12. Bennett CF, Swayze EE. RNA targeting therapeutics: molecular mechanisms of antisense oligonucleotides as a therapeutic platform. Annu Rev Pharmacol Toxicol. 2010;50:259-293.

13. $\mathrm{Li} \mathrm{H}$, et al. Transferrin therapy ameliorates disease in beta-thalassemic mice. Nat Med. 2010;16(2):177-182.

14. Schmidt PJ, et al. An RNAi therapeutic targeting Tmprss6 decreases iron overload in $\mathrm{Hfe}^{-/-}$mice and ameliorates anemia and iron overload in murine beta-thalassemia intermedia. Blood. 2013; 121(7):1200-1208.

15. Sisay MT, et al. Identification of the first low-molecular-weight inhibitors of matriptase-2. J Med Chem. 2010;53(15):5523-5535.

16. Bacon BR, Adams PC, Kowdley KV, Powell LW, Tavill AS. Diagnosis and management of hemochromatosis: 2011 practice guideline by the American Association for the Study of Liver Diseases. Hepatology. 2011;54(1):328-343.

17. Kwiatkowski JL. Real-world use of iron chelators. Hematology Am Soc Hematol Educ Program. 2011; 2011:451-458.

18. Weatherall DJ. The definition and epidemiology of non-transfusion-dependent thalassemia. Blood Rev. 2012;26(suppl 1):S3-S6. 